\section{QTL mapping for microtuber ABA content in a diploid potato population}

\author{
Raja Mohib Muazzam Naz ${ }^{1,2}$, Safia Ramzan ${ }^{1}$, Gege $\mathrm{Li}^{1}$, Mengtai \\ $\mathrm{Li}^{1}$, Jun Liu ${ }^{1}$, Xingkui Cai ${ }^{1 *}$ and Conghua $\mathrm{Xie}^{1}$
}

\begin{abstract}
Abscisic acid $(A B A)$ is known to impact many areas of plant growth and development and is also assumed to facilitate plant stress regulation. $A B A$ is also involved in the rapid suberization of wounds and is the essential dormancy release regulator. The direct application of DNA markers to plant populations enables the use of mapping to help determine the regulation of a quantitatively inherited trait isolated in a population. QTLs represent a chromosomal region that is linked to a marker gene and that significantly affects the quantitative trait under review. In the present study, we investigated the ABA content after harvest and after sprouting in a diploid population. The most noticeable QTLS related to $A B A$ were found on chromosomes I and IV, and these QTLS fully explained $6.5 \%$ and $7 \%$ of the entire phenotypic variance, respectively. The acquired information advances our understanding of the inheritance of traits applicable for variety development.
\end{abstract}

Keywords: $A B A$, microtuber, potato, quantitative trait loci, dormancy.

\section{INTRODUCTION}

As the single most important non-cereal crop species, potato (Solanum tuberosum L.) is grown in approximately 150 countries and is an important part of the global food system. The contribution of potatoes in the developing world for nutrition and food security and for the elimination of hunger and malnutrition is equal to that of rice, wheat, and maize but with a higher yield ratio (Shrestha et al. 2018)

In potato tubers, the dormancy period, also known as the resting period, represents the physiological condition when the tubers are incapable of sprout growth (Bisognin et al. 2018); this is true even if conditions are favorable (i.e., darkness, high humidity and warm temperature) for growth (Sonnewald and Sonnewald 2014). A long dormancy period guarantees that the quality of stored tubers can be preserved for up to 7 months after harvest (Tarn et al. 1992). In the commercial sector for more than 40 years, chemical inhibitors have been used to control sprouting (Van den Berg et al. 1996). The dormancy period of a tuber can also be prolonged by storing tubers at low temperatures approximately $4^{\circ} \mathrm{C}$. This method, however, is not desirable for processing companies since nonreducing sugars are converted into reducing sugars (Li et al. 2018). Nonetheless, an increasing number of consumers prefer potatoes without chemical residues. Long dormancy has increasingly become important in cultivars (Tarn et al. 1992). Alternatively, the dormancy period can be
Crop Breeding and Applied Biotechnology 20(2): e20622021, 2020 Brazilian Society of Plant Breeding. Printed in Brazil http://dx.doi.org/10.1590/198470332020v20n2a17

\author{
*Corresponding author: \\ E-mail: caixingkui@mail.hzau.edu.cn \\ (D) ORCID: 0000-0002-0336-8375 \\ Received: 24 January 2018 \\ Accepted: 29 April 2018 \\ Published: 24 March 2020
}

${ }^{1}$ Ministry of Agriculture, Huazhong Agricultural University, National Center for Vegetable Improvement, Key Laboratory of Potato Biology and Biotechnology, Wuhan 430070, China

${ }^{2}$ University of Poonch Rawalakot, Faculty of Agriculture, Department of Horticulture, Azad Jammu \& Kashmir-Pakistan 
prolonged genetically. Studies have revealed long dormancy periods for select South American tuber-bearing potato relatives with diploid cells (Hermundstad and Peloquin 1985); genes from this species can be transferred into the gene pool of cultivated potato.

$A B A$ is referred to as the regulator of fundamental dormancy release. The role of $A B A$ in dormancy tends to occur throughout the development of the seed (Yamaguchi et al. 2018). Investigations of transgenic and mutant plants offers solid indications that $A B A$ biosynthesis as well as responses to ABA-like phytohormones is complex during the maintenance and onset of dormancy. Additionally, grafting trials and mutual crosses between wild-type plants and ABA biosynthetic mutants have demonstrated that dormancy in developing seeds is dependent on ABA that is produced by the embryo and not on $A B A$ that is produced by the maternal parent of the offspring (Frey et al. 2004).

Abiotic stress is an essential threat to agricultural production. Plant development and growth and thus yield can be seriously suppressed by stress conditions. As such plants have evolved different types of defense mechanisms to cope with stress, though they are not sufficient for protection. ABA is regarded as an isoprenoid phytohormone that facilitates the regulation of different physiological processes, which further extends to protein storage and stomatal opening and additionally gives adaptations to numerous stresses such as salinity, cold and drought. ABA is also known as a vital messenger that operates as the signaling mediator for the regulation of the adaptive reactions of plants to various environmental stresses (Sah et al. 2016).

The regulatory processes of wound healing and tuber dormancy are controlled by ABA in potato tubers (Suttle et al. 2012). During tuber dormancy, the overall content of $A B A$ is at its highest level; at the instant after harvest, the dormancy of the meristem is the deepest. During the storage period, as dormancy diminishes, the levels of ABA decrease. Despite significant differences in the dormancy period among tuber cultivars, the relation between dormancy progression and ABA levels has been shown in various potato cultivars under different storage conditions (Biemelt et al. 2004). The use of chemical inhibitors to induce a reduction in the levels of $A B A$ in tubers, for example, using fluridone, leads to premature growth sprouting, therefore showing the need for sustained synthesis of ABA in the maintenance of tuber dormancy. On the other hand, exogenous ABA application generally causes minimal and short-lived sprouting effects on tubers. ABA easily breaks down; hence, exogenous ABA's inability to stop the development of sprouts for a longer period of time is most likely because of its rapidly occurring metabolic activity (Destefano-Beltrán et al. 2006).

According to Mackay et al. (2009), in terms of quantitative hereditary qualities, QTL mapping is regarded as a standard strategy. QTL mapping ordinarily originates with the gathering of phenotypic and genotypic data obtained from a segregating population, accompanied by statistical analysis of all conceivable marker loci in which allelic variation is linked to the phenotype. Consequently, this process allows only for estimated QTL mapping and is normally referred to as primary QTL mapping. The techniques and methodologies for primary QL mapping are well established (Hackett 2002).

The physiology of microtubers closely resembles the physiology of field-grown potatoes. Some literature data indicate that microtuber systems may be good for determining various processes due to their convenience, reliability, ease of handling, and season independency (Coleman et al. 2001, Donnelly et al. 2003)

Potato breeding programs constantly need new approaches to improve efficacy by increasing the frequency of selected genotypes and reducing time and costs (da Silva et al. 2006). For commercial viability, modernized processing and table potato cultivars have to meet strict stipulations with respect to tuber quality characteristics. As reviewed in this research study, ABA content is very important in the regulation of tuber dormancy and in the healing of wounds. By QTL analysis of a segregating population with a stringent microtuber system, the present study intended to identify the genetic loci controlling potato tuber $A B A$ biosynthesis and to provide a potential opportunity for genetic manipulation and selection of ABA-related traits.

\section{MATERIAL AND METHODS}

\section{Plant material}

The diploid potato $(2 n=2 x=24) F_{1}$ population in this study consisted of 178 individuals from a cross between the cultivated species Solanum phureja acc. DM1-3 516 R44 and the wild species S. Chacoense acc. PI 320285. The population 
is the same as that used in our previous research (Naz et al. 2018), and the female parent, DM1-3 516 R44, which was used to develop the reference potato genome sequence (Potato Genome Sequencing Consortium 2011), is a homozygous line with short dormancy developed through anther culture and somatic doubling. The male parent PI 320285 has a long dormancy period. The hybrids were cultivated in vitro on MS media (Murashige and Skoog 1962) supplemented with $4 \%$ sucrose and $0.7 \%$ agar at $20 \pm 1{ }^{\circ} \mathrm{C}$ under a $16 / 8$ hour light/dark cycle and $83 \mu \mathrm{moL} \mathrm{m} \mathrm{m}^{-1}$ of light intensity.

\section{Microtuberization}

For microtuberization same procedure was adopted as described previously (Naz et al. 2018). The experiment was conducted in 2014 and repeated in 2015.

\section{Phenotyping}

\section{ABA Extraction, Purification, and Quantification}

The ABA content was determined for the tubers sampled after harvest (one week after harvest [AH]) and after sprouting (when the first bud had a length of $2 \mathrm{~mm}[A S]$ ). The conditions of microtuber harvest, storage for sprouting and ABA (extraction, purification and quantification) were the same as those in our previous study (Naz et al. 2018).

\section{DNA extraction, marker analysis and map construction}

Fresh leaves of the in-vitro grown plantlets were utilized for DNA extraction in accordance with the CTAB protocol as described by Dellaporta et al. (1983). The F1 population was further genotyped by polymerase chain reaction for approximately 174 segregating marker alleles distributed across all 12 potato chromosomes, as described previously (Naz et al. 2018). The MapQTL 6 application program (software) was utilized for the analysis of the marker-QTL relationship by the use of the composite interval mapping method (CIM method). The map comprised approximately 174 marker alleles acquired from $S$. chacoense. The molecular and phenotypic marker data were set up as indicated by the instructions provided in the manuals (Van Ooijen 2006, 2009). In the same manner, the proportion of notable phenotypic variance attributable to a QTL of interest was computed by the coefficient of determination $\left(R^{2}\right)$ directly from a regression model, which is essential for analysis (Van Ooijen 2006, 2009). In addition, permutation test 1000 iterations, with $\mathrm{P}<0.05$ were executed to estimate the logarithm of odds (LOD) to verify the QTL. The LOD score and location graphs of the QTLs were developed with the use of MapChart 2.2 software (Voorrips 2002).

\section{RESULTS}

\section{Phenotypic variation}

Significant differences in ABA content existed among genotypes of the segregating population under the same in vitro conditions. The frequency distribution of ABA showed typical quantitative variation, as they were continuously distributed (Figure 1). The trait values observed in the mapping population were consistent across the two

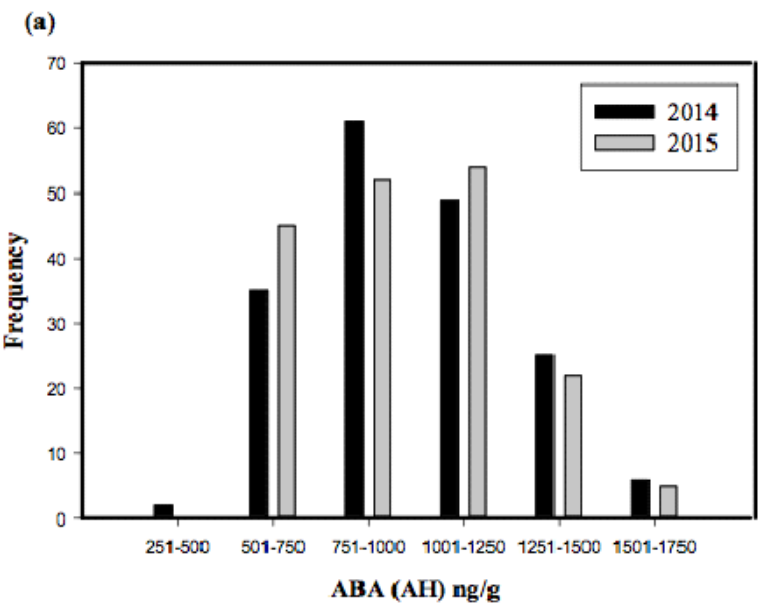

(b)

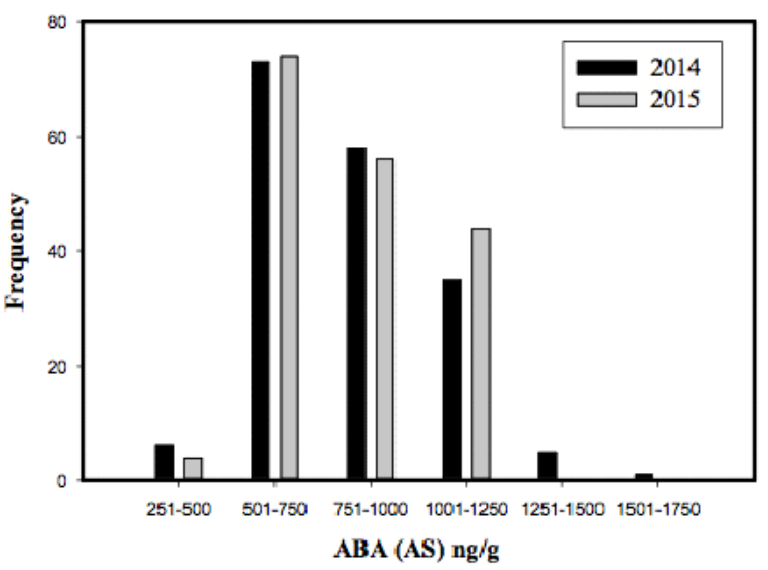

Figure 1. Distributions of phenotype of (a) ABA content $(A H)$ and (b) ABA content (AS) measured in 2014 and 2015 of 178 genotypes. 
consecutive years, as indicated by the significant Pearson correlation coefficient. The correlation coefficient for ABA (AH) was as follows: $r=0.948(P<0.0001)$. However, a weaker correlation ( $r=0.866, P<0.0001)$ was observed for ABA after sprouting (AS), which might be due to abrupt changes among genotypes after sprouting (Figure 2).

\section{QTL Mapping}

Based on the results from the permutation test, a LOD score of 2.5 was declared the QTL significance threshold at the $95 \%$ confidence level. A QTL associated with ABA $(A H)$ and $A B A(A S)$, explaining 6.3 to $7 \%$ of the phenotypic variation, was identified on CHRs I and IV in the linkage map (Figure 3, Table 1). The LOD scores of significant QTLs ranged from 2.50 to 2.82 .

The QTLs for ABA (AH) were mapped to regions on CHRs I and IV, with the maximum effect close to the markers S198_140 and S093_170 (Figure 3). A strong QTL with a LOD score of 2.82 was identified for ABA (AH) in 2014, which explained $7 \%$ of the phenotypic variation. The QTLs for ABA (AS) were also mapped to the regions on CHRs I and IV, with the maximum effect close to the marker PGSC21436_160 (Figure 3). A strong QTL with a LOD score of 2.57 was identified for ABA (AS) in 2015, which explained $6.4 \%$ of the phenotypic variation.

The QTLs for ABA (AH) and (AS) for 2014 and 2015 were not exactly located in the same region. In 2014, the QTLs for ABA (AH) were located on CHRs I, IV and IV in 2015; however, the QTLs for ABA (AS) in 2014 were located on CHRs IV and I in 2015 (Figure 3).

\section{DISCUSSION}

$A B A$ is known to be the most important trait for the regulation of physiological and developmental function in plants. Among all the essential functions related to healing processes of injured periderm tissue in potato, suberization (a healing process) has not thoroughly studied in terms of its genetic basis, and an understanding of the regulators that accelerate the processes involved is lacking. The physiology of injury healing (suberization) as well as injury-related imperfections is of global significance in light of the degree of food and financial losses hindered by these processes. These outcomes suggest a conceivable genomic region bestowing suberization because ABA accelerates the suberization of injured potato.

Table 1. QTL related to dormancy, $A B A(A H)$ and $A B A(A S)$ on map of the diploid segregating population. For each QTL, chromosome (CHR), peak position, LOD and locus at top are provided

\begin{tabular}{|c|c|c|c|c|c|c|c|}
\hline Trait & CHR & Peak position & Locus at peak & LOD & Interval (2-LOD) & PVE (\%) & Additive effect \\
\hline \multirow[t]{3}{*}{ ABA(AH) 2014} & 1 & 38.73 & PGSC21436_160 & 2.59 & $33.4-45.6$ & 6.5 & -71.05 \\
\hline & IV & 0.00 & S198_140 & 2.82 & $0-32.1$ & 7 & -71.50 \\
\hline & IV & 0.07 & S093_170 & 2.82 & $0.07-32.1$ & 7 & -71.50 \\
\hline$A B A(A H) 2015$ & IV & 0.07 & S093_170 & 2.5 & $0.07-34.1$ & 6.3 & -65.78 \\
\hline ABA(AS) 2014 & IV & 19.795 & ssr_68852 & 2.51 & $5.2-36.1$ & 6.3 & -56.68 \\
\hline ABA(AS) 2015 & I & 38.732 & PGSC21436_160 & 2.57 & $31.1-45.6$ & 6.4 & -53.64 \\
\hline
\end{tabular}


CHR I

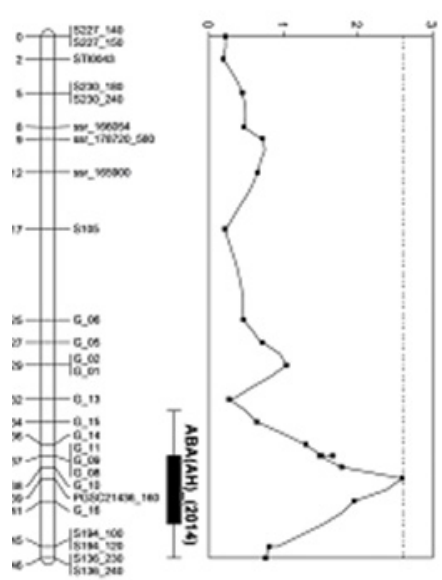

(a)
CHIR IV

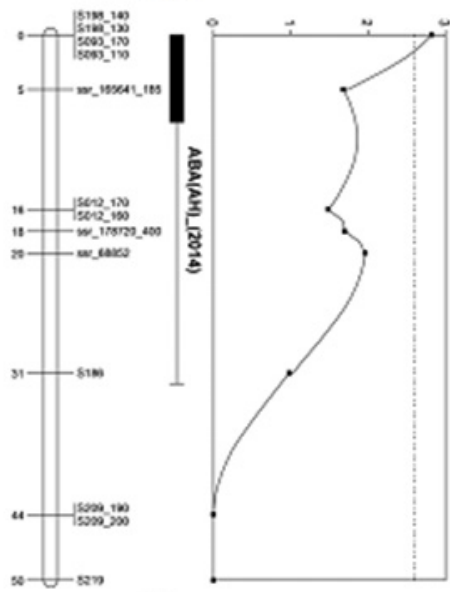

(b)

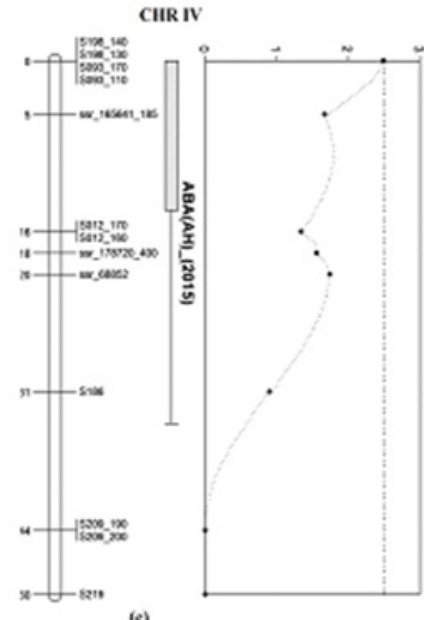

(e)
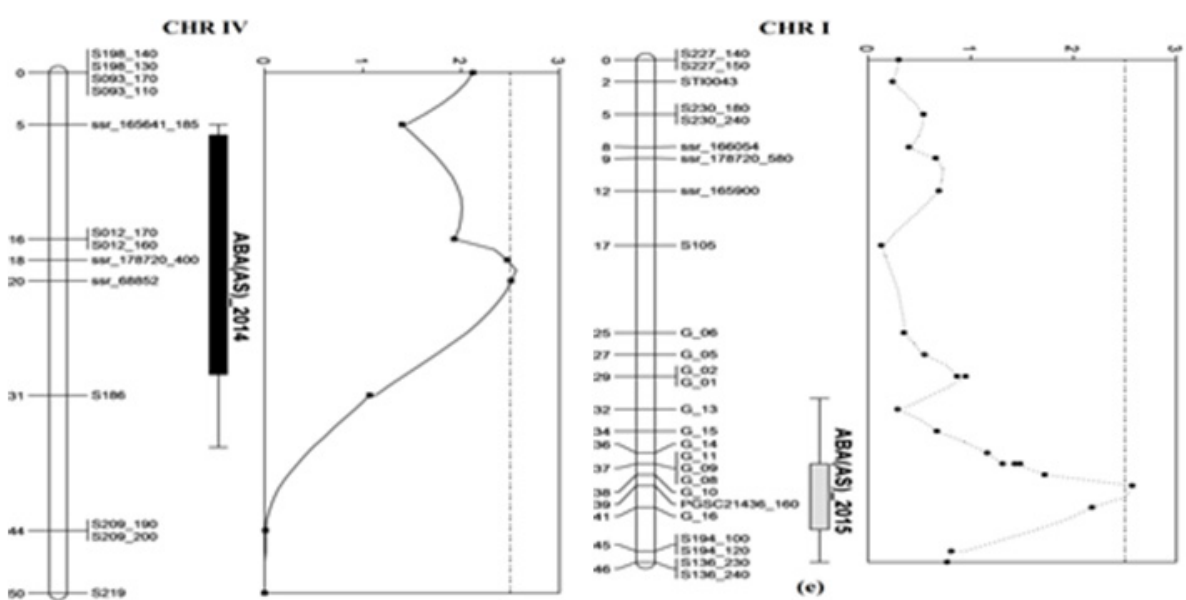

(d)

Figure 3. Location and LOD graphs of the QTLs identified by composite interval mapping (CIM) on CHR I and IV (a) ABA (AH) 2014, (b) ABA (AH) 2014, (c) ABA(AH) 2015), (d)ABA(AS) 2014 and, (e)ABA(AS) 2015. Locations of one QTL (black bars) denotes 2014 and (light gray bars) denotes 2015. The bar indicates the 2-LOD support interval of the QTL. $95 \%$ thresholds (vertical solid black Lines for the year2014) and (vertical dotted Lines for the year 2015) are determined by permutation tests on each QTL.

In addition, $\mathrm{ABA}$ is referred to as a vital messenger acting as a signaling mediator for the regulation of the plant adaptive response to various conditions of environmental stress. Previous studies have revealed that the dormancy breakage relative to after-ripening evidently is distinctly different from ABA catabolism, resulting in a decrease in the content of ABA in the embryo as well as a reliable increase in inert ABA metabolites such as dihydrophaseic acid (DPA) and phaseic acid (PA) (Zeevaart 1977).

Furthermore, the objectives of potato breeding at the breeding stage have resulted in increased numbers of cultivars that are not just proper for various purposes but also resistant to abiotic stresses, such as chilling, high temperature, drought, and salinity, which are the main restrictors in potato production. In this study, we assessed the important physiological traits of ABA at the moment harvest and the sprouting process had completed. ABA performs important, widespread functions in the regulation of the release of primary dormancy; it controls the ripening and growth processes, which are important in suberization and abiotic stress responses (injury healing of potato tuber) during harvest.

Because of the diversified roles of ABA in the production of potato, we assessed QTLs influencing this important quality as well as the physiological traits that were further tagged with the use of the genetic map. In total, 2 QTLs for 
Table 2. Information related to ABA specific genes

\begin{tabular}{lcccc}
\hline Accession no. & CHR & Protein name & Function & Superfamily \\
\hline pfam02496 & IV & $\begin{array}{c}\text { ABA/WDS induced } \\
\text { protein }\end{array}$ & $\begin{array}{c}\text { This is a family of plant proteins induced by } \\
\text { water deficit stress (WDS), or abscisic acid } \\
\text { (ABA) stress and ripening. }\end{array}$ & cl03622 \\
cl03622 & IV & $\begin{array}{c}\text { ABA/WDS induced } \\
\text { protein }\end{array}$ & $\begin{array}{c}\text { This is a family of plant proteins induced by } \\
\text { water deficit stress (WDS), or abscisic acid } \\
\text { (ABA) stress and ripening. }\end{array}$ & NCBI database \\
\hline
\end{tabular}

$A B A$ after harvesting $(A H)$ as well as after sprouting (AS) were identified. The results obtained demonstrated a critical relationship between $A B A(A H)$ and $A B A(A S)$, and 6 markers were found. Additionally, similar QTL positions for ABA (AH) in 2014 and 2015, as well as for ABA (AS) in 2014, were distinguished on CHR IV on the genetic map. With respect to the existence of prominent segregation and trait diversity, the segregation of the population is reasonable for the recognition of marker-trait relationship QTLs for studying $A B A(A H)$ and $A B A(A S)$.

Simko et al. (1997) conducted a study on the variation related to abscisic acid (ABA), particularly in mapping populations. In the present study, a minimum of three loci on approximately three chromosomes (VII, IV, and II) were associated with the ABA content. Moreover, there was no relationship between dormancy and ABA content, which could be determined by comparing epistatic interactions and QTL positions. Subsequent studies have shown that in the mapping of additional QTLs for hormones (ABA, polyamines, zeatin riboside, tuberonic acid glucoside, and gibberellin $A_{1}$ ), a few of these QTLs coincide with the QTL for dormancy. On the basis of the results obtained, we additionally discovered that the results for the QTLs for dormancy present on CHRs I and IV are similar to the results of Ewing et al. (2004) and Simko et al. (1997). We mapped the QTL controlling ABA content AH in 2014 and AS in 2015 to the same location for dormancy on CHR I ( $N a z$ et al. 2018); it can thus be concluded that ABA biosynthesis controls the dormancy of potato tubers. Nonetheless, these physiological research studies require more substantiation to demonstrate that $A B A$ genetically influences tuber dormancy. The function of ABA is wide-ranging and regulates numerous traits, which may explain why we discovered QTLs within various chromosomal regions.

To identify the specific genes involved we selected the identified QTL region from position 58999991 to 60999960 on chromosome IV. By using the NCBI database, we determined that the length of the region was approximately 1999970 bp (1.91 Mb). We found approximately 276 genes in this region by using Softberry software based on the DM1-3 516 R44 sequence. There were 2 candidate genes found in the identified region on chromosome IV linked to specific gene accession Nos. pfam02496 and cl03622 (Table 2). These genes encode proteins that are members of a family of plant proteins induced by water deficit stress (WDS) or by abscisic acid (ABA) and ripening. For CHR I, we selected the identified QTL region from marker G_13 to S194. By using the NCBI database, we determined that the length of the region was approximately 1,452,694 bp (1.45 Mb). We found approximately 217 genes in this region by using Softberry software based on the DM1-3 516 R44 sequence. Of these genes, 131 control different traits. Unfortunately, none of these genes were related to our trait of interest $(A B A)$. However, the function of the remaining 86 genes is still unknown.

In the results we obtained, the QTLs contributing to ABA AH in 2014 and 2015 as well as AS in 2014 mapped to nearly the same location on CHR IV, as shown in figure 3. This may consequently have occurred because of outbreeding; hereditary qualities of cultivated potato as well as any chosen characteristic to potato breeders are truly polygenic in nature, having an extraordinary environmental factor, as indicated by Mullins et al. (2006). Additionally, studies such as those involving fine mapping will be needed to determine whether there is an ABA causal relationship with related traits, for example, suberization (injury healing) together with dormancy and abiotic stress. In this way, ABA appears to activate specific major enzymes, which could most likely be active in suberization, according to Cottle and Kolattukudy (1982).

\section{CONCLUSION}

Amazingly, 95\% of the increasing global population is situated in developing nations with persistently increasing factors that tend to be increasing the pressure against and the scarcity of agricultural resources. In this manner, to satisfy the demand, the production of food has to increase. Thus, an understanding of abiotic stress tolerance of plants 
has vital and widespread consequences that are difficult to determine. Furthermore, ABA is an indispensable hormone and operates as a focal regulator of the response to various plant stresses, such as salinity, low temperature, drought, the process of suberization (injury healing) and the regulation of dormancy. In light of the advancement of molecular mapping, distinguishing the genetic factors that regulate the ABA content prior and after potato tuber sprouting can assist in the breeding of different genotypes. Prospective studies should emphasize the methods of an arrangement relative to genomic as well as genetic ways to identify the genetic element regulating the content of ABA in nondormant and dormant seeds. This is an approach that offers opportunities to coordinate the persistent breeding of fundamental genotypic mechanisms related to ABA content, providing steady yields in the face of abiotic stress.

\section{ACKNOWLEDGMENTS}

This project was financially supported by the Key Project of the Natural Science Foundation of Hubei Province (2015CFA104), the Special Fund for Agro-scientific Research in the Public Interest from the Ministry of Agriculture, China (201303007), and the Special Fund for Agro-scientific Research in the Public Interest, Hubei (2013BBB07).

\section{REFERENCES}

Biemelt S, Tschiersch H and Sonnewald U (2004) Impact of altered gibberellin metabolism on biomass accumulation, lignin biosynthesis, and photosynthesis in transgenic tobacco plants. Plant Physiology 135: 254-265.

Bisognin DA, Manrique-Carpintero NC and Douches DS (2018) QTL analysis of tuber dormancy and sprouting in potato. American Journal of Potato Research 95: 374-382.

Coleman WK, Donnelly DJ and Coleman SE (2001) Potato microtubers as research tools: a review. American Journal of Potato Research 78: 47-55.

Cottle W and Kolattukudy PE (1982) Abscisic acid stimulation of suberization: induction of enzymes and deposition of polymeric components and associated waxes in tissue cultures of potato tuber. Plant Physiology 70: 775-780.

Dellaporta SL, Wood J and Hicks JB (1983) A plant DNA minipreparation: version II. Plant Molecular Biology Reporter 1: 19-21.

Destefano-Beltrán L, Knauber D, Huckle L and Suttle J (2006) Chemically forced dormancy termination mimics natural dormancy progression in potato tuber meristems by reducing $A B A$ content and modifying expression of genes involved in regulating $A B A$ synthesis and metabolism. Journal of Experimental Botany 57: 2879-2886.

Donnelly DJ, Coleman WK and Coleman SE (2003) Potato microtuber production and performance: a review. American Journal of Potato Research 80: 103-115.

Ewing EE, Simko I, Omer EA and Davies PJ (2004) Polygene mapping as a tool to study the physiology of potato tuberization and dormancy. American Journal of Potato Research 81: 281-289.

Frey A, Godin B, Bonnet M, Sotta B and Marion-Poll A (2004) Maternal synthesis of abscisic acid controls seed development and yield in Nicotiana plumbaginifolia. Planta 218: 958-964.

Hackett CA (2002) Statistical methods for QTL mapping in cereals. Plant Molecular Biology 48: 585-599.
Hermundstad SA and Peloquin SJ (1985) Germplasm enhancement with potato haploids. Journal of Heredity 76: 463-467.

Li J, Huang W, Cao H, Xiao G, Zhou J, Xie C, Xia J and Song B (2018) Additive and epistatic QTLS underlying the dormancy in a diploid potato population across seven environments. Scientia Horticulturae 240: 578-584.

Mackay TF, Stone EA and Ayroles JF (2009) The genetics of quantitative traits: challenges and prospects. Nature Reviews Genetics 10: 565-577.

Mullins E, Milbourne D, Petti C, Doyle-Prestwich BM and Meade C (2006) Potato in the age of biotechnology. Trends in Plant Science 11: 254-260.

Murashige T and Skoog F (1962) A revised medium for rapid growth and bio assays with tobacco tissue cultures. Physiologia plantarum 15: 473-497.

Naz RMM, Li M, Ramzan S, Li G, Liu J, Cai X and Xie C (2018) QTL mapping for microtuber dormancy and $\mathrm{GA}_{3}$ content in a diploid potato population. Biology Open 7: bio027375.

Potato Genome Sequencing Consortium (2011) Genome sequence and analysis of the tuber crop potato. Nature 475: 189-195.

Sah SK, Reddy KR and Li J (2016) Abscisic acid and abiotic stress tolerance in crop plants. Frontiers in Plant Science 7: 571.

Shrestha KP, Adhikari SP and Yadav S (2018) Economics of potato production in rural area of Ilam district, Nepal. International Journal of Applied Sciences and Biotechnology 6: 344-350.

Silva GO, Souza VQ, Pereira AS, Carvalho FIF and Fritsche Neto R (2006) Early generation selection for tuber appearance affects potato yield components. Crop Breeding and Applied Biotechnology 6: 73-78.

Simko I, McMurry S, Yang HM, Manschot A, Davies PJ and Ewing EE (1997) Evidence from polygene mapping for a causal relationship between potato tuber dormancy and abscisic acid content. Plant Physiology 115: 1453-9.

Sonnewald S and Sonnewald U (2014) Regulation of potato tuber sprouting. Planta 239: 27-38. 
Suttle JC, Abrams SR, De Stefano-Beltrán L and Huckle LL (2012) Chemical inhibition of potato ABA-8'-hydroxylase activity alters in vitro and in vivo $A B A$ metabolism and endogenous $A B A$ levels but does not affect potato microtuber dormancy duration. Journal of Experimental Botany 63: 5717-5725.

Tarn TR, Tai GC, De Jong H, Murphy AM and Seabrook JE (1992) Breeding potatoes for long-day, temperate climates. Plant Breeding Reviews 9: 217-332.

Van den Berg JH, Ewing EE, Plaisted RL, McMurry S and Bonierbale MW (1996) QTL analysis of potato tuber dormancy. Theoretical and Applied Genetics 93: 317-324.

Van Ooijen JW (2006) JoinMap ${ }^{\circledR}$ 4, software for the calculation of genetic linkage maps in experimental populations. Kyazma BV, Wageningen, https://www.kyazma.nl/index.php

Van Ooijen JW (2009) MapQTL ${ }^{\circledR}$ 6, software for the mapping of quantitative trait loci in experimental populations of diploid species. Kyazma BV, Wageningen, https://www.kyazma. nl/index.php/MapQTL/Manual/.

Voorrips RE (2002) MapChart: software for the graphical presentation of linkage maps and QTLs. Journal of Heredity 93: 77-78.

Yamaguchi S, Kamiya Y and Nambara E (2018) Regulation of ABA and $G A$ levels during seed development and germination in Arabidopsis. Annual Plant Reviews 27: 224-247.

Zeevaart JA (1977) Sites of abscisic acid synthesis and metabolism in Ricinus communis L. Plant Physiology 59: 788-791. 